\title{
Binocular Disparity Selectivity Weakened after Monocular Deprivation in Mouse V1
}

\author{
(DBenjamin Scholl, ${ }^{1 *}$ ๑) Jagruti J. Pattadkal, ${ }^{2 *}$ and Nicholas J. Priebe ${ }^{2}$ \\ ${ }^{1}$ Max Planck Florida Institute, Jupiter, Florida 33458, and ${ }^{2}$ Center for Perceptual Systems, Department of Neuroscience, College of Natural Sciences, \\ University of Texas at Austin, Austin, Texas 78712
}

Experiences during the critical period sculpt the circuitry within the neocortex, leading to changes in the functional responses of sensory neurons. Monocular deprivation (MD) during the visual critical period causes shifts in ocular preference, or dominance, toward the open eye in primary visual cortex (V1) and disrupts the normal development of acuity. In carnivores and primates, MD also disrupts the emergence of binocular disparity selectivity, a cue resulting from integrating ocular inputs. This disruption may be a result of the increase in neurons driven exclusively by the open eye that follows deprivation or a result of a mismatch in the convergence of ocular inputs. To distinguish between these possibilities, we measured the ocular dominance (OD) and disparity selectivity of neurons from male and female mouse V1 following MD. Normal mouse V1 neurons are dominated by contralateral eye input and contralateral eye deprivation shifts mouse V1 neurons toward more balanced responses between the eyes. This shift toward binocularity, as assayed by OD, decreased disparity sensitivity. MD did not alter the initial maturation of binocularity, as disparity selectivity before the MD was indistinguishable from normal mature animals. Decreased disparity tuning was most pronounced in binocular and ipsilaterally biased neurons, which are the populations that have undergone the largest shifts in OD. In concert with the decline in disparity selectivity, we observed a shift toward lower spatial frequency selectivity for the ipsilateral eye following MD. These results suggest an emergence of novel synaptic inputs during MD that disrupt the representation of disparity selectivity.

Key words: binocular disparity; critical period; monocular deprivation; mouse visual cortex; plasticity

\section{Significance Statement}

We demonstrate that monocular deprivation during the developmental critical period impairs binocular integration in mouse primary visual cortex. This impairment occurs despite an increase in the degree to which neurons become more binocular. We further demonstrate that our deprivation did not impair the maturation of disparity selectivity. Disparity selectivity has already reached a matured level before the monocular deprivation. The loss of disparity tuning is primarily observed in neurons dominated by the open eye, suggesting a link between altered inputs and loss of disparity sensitivity. These results suggest that new inputs following deprivation may not maintain the precise spatial relationship between the two eye inputs required for disparity selectivity.

\section{Introduction}

Experience-dependent plasticity during the developmental critical period (CP) shapes cortical circuit anatomy and functions (Katz and Crowley, 2002; Espinosa and Stryker, 2012). A prime example of CP plasticity is ocular dominance (OD) plasticity,

\footnotetext{
Received April 8, 2016; revised April 23, 2017; accepted May 9, 2017.

Author contributions: B.S., J.J.P., and N.J.P. designed research; B.S. and J.J.P. performed research; B.S. and J.J.P. analyzed data; B.S., J.J.P., and N.J.P. wrote the paper.

This work was supported by National Institutes of Health Grant EY-025102 and Human Frontiers Science Program. J.J.P. is a Howard Hughes Medical Institute International Student Research Fellow. We thank Jessica Hanover for guidance and helpful discussions.

The authors declare no competing financial interests.

*B.S. and J.J.P. contributed equally to this study.

Correspondence should be addressed to Dr. Nicholas J. Priebe, Department of Neuroscience, University of Texas at Austin, 2400 Speedway, Austin, TX 78712.E-mail: nicholas@mail.utexas.edu.

DOI:10.1523/JNEUROSCI.1193-16.2017

Copyright $\odot 2017$ the authors $\quad 0270-6474 / 17 / 376517-10 \$ 15.00 / 0$
}

where the OD of V1 neurons shifts toward the open eye following monocular deprivation (MD). In primates and carnivores, MD results in both a shift of neurons' responses to being dominated by the open eye and a decline in binocular interactions necessary for 3D representation of the world (Julesz, 1971). The decline in binocular disparity sensitivity is consistent with an increase in neuron monocularity, but whether these two are linked, however, is unclear.

Mouse V1 neuron responses, in contrast, are normally dominated by the contralateral eye. Contralateral MD shifts OD toward the ipsilateral eye, causing a paradoxical increase in binocularity (Gordon and Stryker, 1996). A conventional measure of binocularity, OD, is based on independent stimulation of each eye (Dräger, 1975; Wagor et al., 1980; Hanover et al., 1999; Tagawa et al., 2005; Mrsic-Flogel et al., 2007; Hofer et al., 2009) but provides no information on binocular integration (LeVay and Voigt, 1988). Disparity 
selectivity, the sensitivity to spatial offsets between the two retinal images, requires the integration of both retinal images (Hubel and Wiesel, 1962; Pettigrew et al., 1968; Joshua and Bishop, 1970; Ohzawa and Freeman, 1986; Longordo et al., 2013; Scholl et al., $2013 \mathrm{a}, \mathrm{b})$. Binocular disparity provides a cue for the depth of objects and modulates V1 neuron responses, which exhibit a variety of disparity preferences ranging from near to far (Poggio and Fischer, 1977; Ohzawa and Freeman, 1986; DeAngelis et al., 1995; Cumming and Parker, 1999).

We used this paradoxical increase in binocularity following MD to study how experience shapes binocular integration. We hypothesized that contralateral eye deprivation could affect disparity selectivity in mouse V1 neurons in three ways. Disparity selectivity could increase if the right and left eye signals are balanced by MD (see Fig. $1 A, B$, left). Alternatively, disparity selectivity could decrease if novel circuitry disrupts the precise receptive field alignment between the eyes (see Fig. $1 B$, right). Finally, disparity selectivity could remain the same if compensatory mechanisms counteract these other effects.

We assessed how MD during the CP alters binocular integration using in vivo two-photon calcium imaging (Stosiek et al., 2003; Kerr et al., 2005; Ohki et al., 2005; Garaschuk et al., 2006; Mrsic-Flogel et al., 2007; Golshani and Portera-Cailliau, 2008; Scholl et al., 2015) from layer $2 / 3$ neurons in the binocular zone of mouse V1. We measured OD and binocular disparity selectivity in normal pre-CP animals, post-CP animals, and animals following MD during the CP. In deprived animals, disparity selectivity was decreased compared with normal post-CP animals. Normal binocular integration exists before the MD was performed, as disparity selectivity and OD were similar between pre-CP and post-CP animals. The decline in disparity selectivity was predominately found in neurons receiving substantial input from the nondeprived eye. In concert with this decline, we found evidence for a disruption in the ipsilateral eye receptive field following MD. Together, our data suggest that eye occlusion reshapes synaptic inputs without preserving functionality required for stereoscopic disparity sensitivity.

\section{Materials and Methods}

Physiology. Procedures for two-photon imaging and physiology were based on those previously described (Scholl et al., 2013a, 2015). Experiments were conducted using normal animals $(n=14, \mathrm{P} 25-\mathrm{P} 60)$ or animals with the contralateral eye deprived during the CP $(n=8)$. Both male and female animals were used. The $4 \mathrm{~d} \mathrm{MD}$ was initiated between P28 and P30 (Gordon and Stryker, 1996; Hanover et al., 1999; Tagawa et al., 2005). Mice were anesthetized with intraperitoneal injections of 1000 $\mathrm{mg} / \mathrm{kg}$ urethane and $10 \mathrm{mg} / \mathrm{kg}$ chlorprothixene. Brain edema was prevented by intraperitoneal injection of $10 \mathrm{mg} / \mathrm{kg}$ dexamethasone. Animals were warmed with a thermostatically controlled heat lamp to maintain body temperature at $37^{\circ} \mathrm{C}$. A tracheotomy was performed. The head was placed in a mouse adaptor (Stoelting), and a craniotomy and duratomy were performed over visual cortex. Eyes were kept moist with a thin layer of silicone oil. Primary visual cortex and binocular zone were located and mapped by multiunit extracellular recordings with tungsten electrodes ( $1 \mathrm{M} \Omega$, Micro Probes). The V1/V2 boundary was identified by the characteristic gradient in receptive locations (Dräger, 1975; Métin et al., 1988). Eye drift under urethane anesthesia is typically small and results in a change in eye position of $<2$ degrees per hour (Sarnaik et al., 2014).

Dye loading and in vivo two-photon microscopy. Bulk loading of a calcium-sensitive dye under continuous visual guidance followed previous protocols (Stosiek et al., 2003; Kerr et al., 2005; Ohki et al., 2005; Mrsic-Flogel et al., 2007; Golshani and Portera-Cailliau, 2008; Scholl et al., 2015) in the V1 binocular zone. Dye solution contained $0.8 \mathrm{~mm}$ Oregon Green 488 BAPTA-1 AM (OGB-1 AM, Invitrogen) dissolved in DMSO (Sigma-Aldrich) with 20\% pluronic acid (Sigma-Aldrich) and mixed in a salt solution $(150 \mathrm{~mm} \mathrm{NaCl}, 2.5 \mathrm{~mm} \mathrm{KCl}, 10 \mathrm{~mm}$ HEPES, pH 7.4, all Sigma-Aldrich). Either 40-80 $\mu \mathrm{M}$ AlexaFluor-594 (Invitrogen) or $125 \mu \mathrm{M}$ sulforhodamine 101 (Sigma-Aldrich) was also included for visualization during and immediately after loading. Patch pipettes (tip diameter $2-5 \mu \mathrm{m}$, King Precision Glass) containing this solution were inserted into the cortex to a depth of $250-400 \mu \mathrm{m}$ below the surface with $1.5 \%$ agarose (in saline) placed on top the brain. The solution was carefully pressure injected (100-350 mbar) over 10-15 min to cause the least amount of tissue damage. OGB-1-AM is only weakly fluorescent before being internalized, so the amount of dye injected was inferred through the red dye. To ensure full loading, we waited $1 \mathrm{~h}$ before adding a glass coverslip for imaging. Metal springs were fastened on the attached headplate to place pressure on the glass coverslip and reduce brain pulsations. Fluctuations in calcium fluorescence were collected with a custom-built two-photon resonant mirror scanning microscope (Stosiek et al., 2003; Golshani and Portera-Cailliau, 2008; Scholl et al., 2015) and a modelocked (925 nm) Chameleon Ultra Ti:Sapphire laser (Coherent). Excitation light was focused by a $40 \times$ water objective $(0.8$ numerical aperture, Nikon). Images were obtained with custom software (Labview, National Instruments). A square region of cortex $300 \mu \mathrm{m}$ wide was imaged at $256 \times 455$ pixels. In all experiments, multiple focal planes, separated by $20-25 \mu \mathrm{m}$, were used to collect data, starting $\sim 150 \mu \mathrm{m}$ below the cortical surface. Before each experiment, neuron drift was measured over a 2-3 min period. If drift occurred, then the glass coverslip and agarose were readjusted to stabilize the brain during stimulus protocol (7-10 $\mathrm{min}$ each focal plane).

Stimuli. Visual stimuli were generated by a Macintosh computer (Apple) using the Psychophysics Toolbox (Brainard, 1997; Pelli, 1997) for MATLAB (The MathWorks). Gratings were presented dichoptically using Sony video monitors (GDM-F520) placed $25 \mathrm{~cm}$ from the animal's eyes. The video monitors had a noninterlaced refresh rate of $100 \mathrm{~Hz}$ and a spatial resolution of $1024 \times 768$ pixels, which subtended $40 \times 30 \mathrm{~cm}$ $(58 \times 46$ degrees). The video monitors had a mean luminance of 40 $\mathrm{cd} / \mathrm{cm}^{2}$. Square-wave drifting gratings (38 degree diameter, $0.02-0.04$ spatial frequency, $100 \%$ contrast, $1-4 \mathrm{~Hz}$ temporal frequency) were presented for 2-3 s. Each stimulus was followed by a $3 \mathrm{~s}$ blank (mean luminance) period. Spontaneous activity was measured during blank (mean luminance) periods interleaved with binocular and monocular drifting grating stimuli, all presented in a pseudorandom sequence. Binocular phase differences (disparities) ranged from 0 to 315 degrees. For a subset of experiments, we also measured preferred orientation (grating orientation ranged 0-315 degrees) and spatial frequency sensitivity using gratings (grating orientation 90 degrees) of spatial frequencies that varied between 0.01 and 0.16 cycles/degree. During imaging sessions, each stimulation protocol was repeated 7-10 times at each focal plane. The microscope objective and photomultiplier tubes were shielded from stray light and the video monitors.

Two-photon calcium imaging analysis. Images were analyzed with custom MATLAB software. Cells were identified by hand from structure images based on size, shape, and brightness. Cell masks were generated automatically following previous methods (Nauhaus et al., 2012). Glia were easily avoided due to their different morphology from both OGB-1 AM-filled neurons. Time courses for individual neurons were extracted by summing pixel intensity values within cell masks in each frame. Responses $\left(F_{t}\right)$ to each stimulus presentation were normalized by the response to the gray screen $\left(\mathrm{F}_{\mathrm{o}}\right)$ immediately before the stimulus came on as follows:

$$
\Delta \mathrm{F} / \mathrm{F}=\left(\mathrm{F}_{\mathrm{t}}-\mathrm{F}_{\mathrm{o}}\right) / \mathrm{F}_{\mathrm{o}}
$$

For each stimulus, the mean change in fluorescence $(\Delta \mathrm{F} / \mathrm{F})$ was calculated in a $0.5 \mathrm{~s}$ window of the response, identified by averaging responses to all stimuli and detecting the global peak. Visually responsive cells were identified if at least one monocular and one binocular stimulus response had the following:

$$
\left(\mu_{\text {Stimulus }}-\mu_{\text {Spontaneous }}\right) /\left(\mathrm{SE}_{\text {Stimulus }}+\mathrm{SE}_{\text {Spontaneous }}\right)>1
$$

$\mu_{\text {Stimulus }}$ refers to the mean stimulus-evoked response, $\mu_{\text {Spontaneous }}$ refers to the mean spontaneous activity, $\mathrm{SE}_{\text {Stimulus }}$ is the stimulus-evoked re- 
A

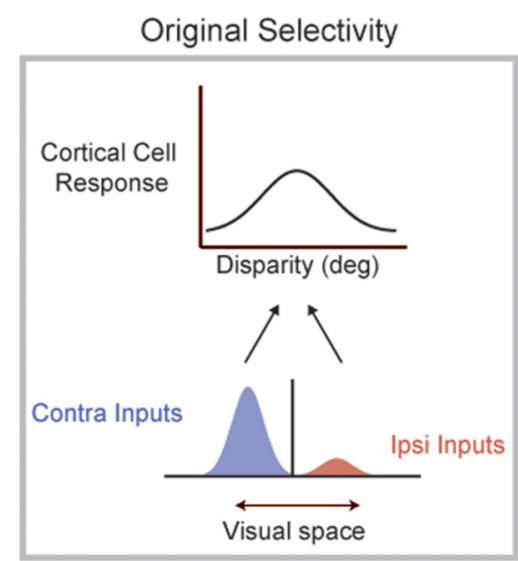

B

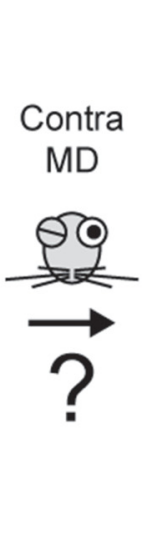

Increased Selectivity

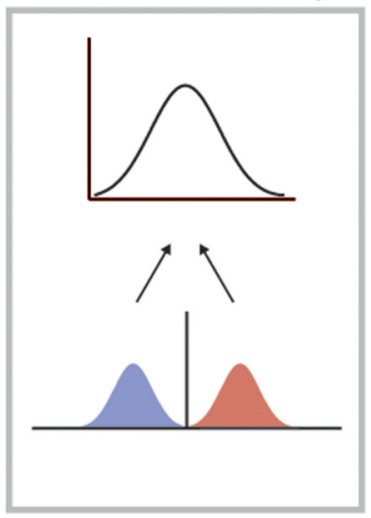

Monocular Deprivation

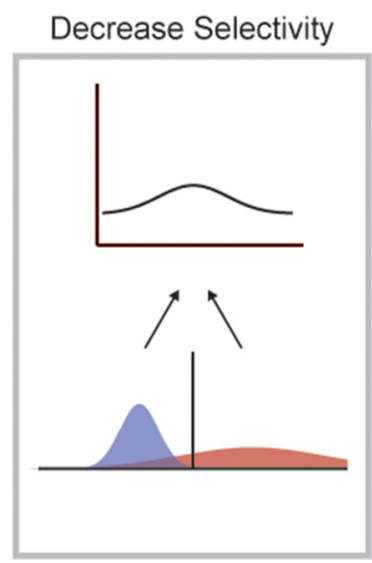

Figure 1. Potential changes to disparity selectivity following MD. A, Normal adult mouse visual cortical neurons are contralaterally biased by monocular stimuli but can be sensitive to binocular disparity by receiving weak ipsilateral inputs. Disparity selectivity formed from the convergence of distributions of contralateral and ipsilateral inputs representing distinct locations in visual space. $B, M D$ of the dominant eye leads to a shift in ocular preference such that neurons are more binocular by monocular stimulation. Increased binocularity could increase disparity selectivity by the enhancement of excitatory input from the weak eye (left). Disparity selectivity could be decreased if the ipsilateral input no longer provides the spatial signal necessary for generating disparity selectivity (right).

sponse $\mathrm{SE}$, and $\mathrm{SE}_{\mathrm{Spontaneous}}$ spontaneous activity SE. Additionally, identified responses to each monocular and binocular stimulus were required have distinct different trial-to-trial fluorescence time courses, so as to not be scaled versions of neuropil activity.

Mean changes in fluorescence from visually responsive neurons were used to generate tuning curves for binocular disparity. Tuning curves in Figures 2 and 3 were fit with a cosine-wave function. To measure OD, we used a standard metric (Cang et al., 2005; Priebe, 2008) as follows:

$$
O D I=\left(R_{\text {ipsi }}-R_{\text {contra }}\right) /\left(R_{\text {ipsi }}+R_{\text {contra }}\right)
$$

Here $R_{\text {contra }}$ and $R_{i p s i}$ represent calcium responses from monocular stimulation of the contralateral and ipsilateral eyes, respectively. Disparity selectivity was quantified using a normalized vector strength (Scholl et al., 2013a, b) as follows:

$$
D S I=\frac{\sqrt{\left(\sum_{\varphi} R_{\varphi} \sin (\varphi)\right)^{2}+\left(\sum_{\varphi} R_{\varphi} \cos (\varphi)\right)^{2}}}{\sum_{\varphi} R_{\varphi}}
$$

Here $\mathrm{R}$ is a calcium response to the particular binocular disparity $(\varphi)$.

Orientation preference was characterized using a double Gaussian curve (Carandini and Ferster, 2000). Both Gaussian curves had the same variance $\left(\sigma^{2}\right)$ but different amplitudes $(\alpha$ and $\beta)$. The second Gaussian curve was constrained to be 180 degree phase shifted from the preferred orientation $\left(\theta_{\text {pref }}\right)$ as follows:

$$
R(\theta)=\alpha e^{-\left(\theta-\theta_{\text {pref }}\right)^{2} /\left(2 \sigma^{2}\right)}+\beta e^{-\left(\theta-\theta_{\text {pref }}+\pi\right)^{2} /\left(2 \sigma^{2}\right)}+k
$$

Here $\mathrm{R}(\theta)$ is the peak calcium response at the particular orientation used $(\theta)$ and $k$ is the baseline spontaneous activity. The preferred orientation was estimated from this fitted equation. The orientation selectivity index was computed using the following equation: (Ringach et al., 2002; Tan et al., 2011) as follows:

$$
O S I=\frac{\sqrt{\left(\sum R(\theta) \sin (2 \theta)\right)^{2}+\left(\sum R(\theta) \cos (2 \theta)\right)^{2}}}{\sum R(\theta)}
$$

Spatial frequency tuning responses were fitted using a single Gaussian function (DeAngelis et al., 1993, Van den Bergh et al., 2010) as follows:

$$
R(\theta)=\alpha e^{-\left(\theta-\theta_{\text {pret }}\right)^{2} /\left(2 \sigma^{2}\right)}+k
$$

Here $\mathrm{R}(\theta)$ is the peak calcium response at the particular spatial frequency $(\theta), \alpha$ is the amplitude of the Gaussian, $\theta_{\text {pref }}$ is the preferred spatial frequency, $\sigma^{2}$ is the variance of the Gaussian used, and $k$ is the baseline. The function is Gaussian along a linear axis. The preferred spatial frequency was estimated from the fitted equation. We also measured spatial resolution, which was defined as the highest spatial frequency at which the fitted tuning curve reached baseline level (Van den Bergh et al., 2010).

Experimental design and statistical analysis. The specific tests used to determine statistical significance are noted in Results. For Gaussian distributions, the $t$ test was used. We determined whether significant differences existed for non-Gaussian distributions using the Mann-Whitney test.

All animal procedures were approved by the University of Texas at Austin Institutional Animal Care and Use Committee.

\section{Results}

We used in vivo two-photon calcium imaging to measure the OD and binocular disparity tuning of neurons in V1 of anesthetized normal and monocularly deprived (MD) mice (see Materials and Methods). Deprivation of the contralateral eye was initiated during the CP (P28-P30) and lasted for $4 \mathrm{~d}$ (Gordon and Stryker, 1996; Hanover et al., 1999; Tagawa et al., 2005). In each experiment, the binocular zone of V1 was identified from extracellular recordings, receptive fields were carefully mapped in a targeted region within the central 30 degrees of the visual field, and a mirror was placed in front of the contralateral eye to allow for presentation of a dichoptic stimulus (Scholl et al., 2013a).

\section{Potential effects of MD on binocular integration}

Cortical neurons in the binocular zone of mouse V1 are normally dominated by input from the contralateral eye. Despite the imbalance in contralateral and ipsilateral eye inputs, prominent disparity selectivity exists in these neurons (Fig. 1A). In this illustration, the receptive field profiles of each eyes are spatially disparate. This spatial disparity of the receptive fields between the two eyes causes certain stimulus phase differences to evoked greater response than others. By probing a range of stimulus phase differences, it is possible to measure the sensitivity of cortical cell for binocular disparity (Ohzawa and Freeman, 1986). 
Contralateral eye deprivation during the $\mathrm{CP}$ in mouse $\mathrm{V} 1$ produces a shift in the OD toward more equivalent inputs between the two eyes (Gordon and Stryker, 1996; Hanover et al., 1999; Tagawa et al., 2005). To illustrate two possible outcomes from $\mathrm{MD}$, we show loss of contralateral input in conjunction with the addition of new ipsilateral input (Fig. 1B). In the first case, binocular disparity selectivity could be increased if the new ipsilateral synaptic inputs have the same receptive field locations as that of preexisting ones (Fig. 1B, left). This is because the preferred interocular phase difference would be preserved and synapses of equivalent strength from each eye will generate larger response modulation with binocular disparity (Scholl et al., 2013a, b). Alternatively, contralateral deprivation might cause a decrease in disparity selectivity if new ipsilateral inputs alter the spatial receptive field (Fig. 1B, right). While we diagram these changes as impacting the ipsilateral receptive field, similarly disruptive changes could occur in the contralateral receptive field during deprivation. Changes in the receptive field brought on by new inputs from either eye could degrade the precise alignment of contralateral and ipsilateral inputs, leading to decreased disparity sensitivity.

\section{Binocular response properties in normal and deprived animals}

To measure monocular responses and binocular disparity selectivity, we pseudorandomly presented dichoptic drifting vertical gratings to each eye alone or simultaneously while recording changes in calcium fluorescence. By varying the interocular phase difference of the drifting gratings, we probed eight binocular disparities (0-315 degrees). During each experiment, we imaged multiple focal planes below the pial surface. A rotatable objective was used to position the cortical surface normal to imaging plane. Cells were chosen by hand from an OGB-1 AM structure image (Fig. 2A, $B$, right) and an automated algorithm created a mask for denoting pixels to average in each frame (Scholl et al., 2015). Across normal animals used ( $n=10$, P35-P60) we identified a total of 4924 neurons, of which 1191 neurons were visually responsive (for criterion, see Materials and Methods) for vertical monocular stimuli (24\%) and 2505 neurons were visually responsive for binocular stimuli (51\%). Across MD animals used ( $n=7$, P33-P35), we identified a total of 3463 neurons, of which 932 neurons were visually responsive for monocular stimuli (27\%) and 1853 neurons were visually responsive for binocular stimuli (54\%). To probe binocular disparity, we used vertically oriented gratings at a single spatial frequency. Mouse V1 neurons exhibit a "salt and pepper" pattern of orientation preferences and a wide range of spatial frequency selectivity (Ohki et al., 2005; Niell and Stryker, 2008), so the vertically oriented gratings may not be effective at stimulating all visually responsive neurons.

Fluorescence fluctuations from cells in mouse V1 were strongly modulated by binocular disparities, compared with stimulation of either eye alone or the blank (mean luminance) period, similarly to previous reports of spiking activity (Scholl et al., 2013a) and twophoton calcium imaging in cat (Kara and Boyd, 2009) and mouse V1 (Scholl et al., 2015). In an example neuron (Fig. 2A), monocular stimulation of either eye evoked approximately equivalent changes in fluorescence $(5 \%-10 \% \Delta \mathrm{F} / \mathrm{F})$, whereas the preferred disparity $(0$ degree phase difference) evoked even larger fluorescence changes $(\sim 30 \% \Delta \mathrm{F} / \mathrm{F})$ and the null disparity (180 degree phase difference) evoked little change $(\sim 5 \% \Delta \mathrm{F} / \mathrm{F})$. Plotting stimulus-averaged peak calcium responses showed response modulation by disparity and binocular responses that were as strong or stronger than responses to either eye alone (Fig. 2A, see tuning curve). From these neural re- sponses, we computed two metrics: an ocular dominance index (ODI, see Materials and Methods) to compare monocular responses from each eye and a disparity selectivity index (DSI, see Materials and Methods) to quantify response selectivity to binocular phase differences. In this example (Fig. 2A), these metrics depicted a binocular, but contralaterally biased neuron with strong disparity selectivity $(\mathrm{ODI}=-0.32, \mathrm{DSI}=0.51)$. Ipsilaterally biased neurons also exhibit strong disparity selectivity $(\mathrm{ODI}=0.11, \mathrm{DSI}=0.29$; Fig. $2 B$ ).

Across all neurons with visually evoked responses to monocular stimuli, we found a shift in OD between normal and deprived animals. As reported previously from spiking (Gordon and Stryker, 1996; Hanover et al., 1999; Tagawa et al., 2005) and calcium responses (Mrsic-Flogel et al., 2007; Kameyama et al., 2010), the contralateral bias in normal mice (Fig. 2E) shifted toward more equivalent monocular responses and a more uniform distribution of OD (Fig. 2F). Because ODI is a symmetric index, to quantify the difference in these distributions we computed the absolute value of ODI, such that a value of 0 indicates binocular responses and a value of 1 indicated perfectly monocular responses. Neurons from normal animals were more monocular than those from $\mathrm{MD}$ animals (normal: mean $|\mathrm{ODI}|=0.64 \pm 0.34$, $n=1191 ; \mathrm{MD}$ : mean $|\mathrm{ODI}|=0.49 \pm 0.33, n=932 ; p<0.001$, Mann-Whitney test; Fig. 2G).

We next investigated how increases in binocularity following MD affected disparity selectivity. Surprisingly, we found a decrease in DSI in deprived animals compared with those raised normally (Fig. $2 H, I$ ). With normal development, neurons exhibited modest disparity selectivity (median DSI $=0.23$, mean DSI $=0.28 \pm 0.19, n=2505$ ), whereas neurons showed a significant reduction in disparity selectivity following MD (median DSI $=0.17$, mean DSI $=0.21 \pm 0.14, n=1853 ; p<0.001$, Student's two-tailed $t$ test; Fig. $2 J$ ). This loss of disparity selectivity demonstrates a divergence in how MD affects OD and disparity selectivity, suggesting that a rearrangement of inputs occurs that disrupts the match of receptive fields necessary for disparity selectivity.

Although we observe a significant decrease in DSI for deprived animals, we wondered whether this decrease is related to differences in peak binocular responses across our population of individual cells $(5 \%-40 \% \Delta \mathrm{F} / \mathrm{F})$. We therefore compared the degree of disparity selectivity in normal and monocularly deprived animals for groups of cells with similar amplitude binocular responses. When comparing only cells with small $(<10 \%)$, medium $(10 \%-$ $20 \%)$, or large $(>20 \%)$ amplitude peak binocular responses, the difference in disparity selectivity between deprived and normal animals was consistent ( $p<0.001$, Mann-Whitney). We did find that DSI was correlated with peak binocular responses (normal: amplitude $<10 \%$ median DSI $=0.20$, amplitude $>20 \%$ median DSI $=$ 0.43 ; MD: amplitude $<10 \%$ median DSI $=0.15$, amplitude $>20 \%$ median DSI $=0.37$ ). This relationship was also monotonic, suggesting that saturation of the calcium indicator was unlikely to influence our measurements of DSI. Another potential issue is that we used vertical gratings, while the orientation preference of individual cells varies substantially. In a subset of experiments, we separated neurons into those preferring vertical and horizontal gratings and did not find a difference in DSI (preferred orientation $=90 \pm 45^{\circ}, n=$ 27 , median DSI $=0.28$; preferred orientation $=0 \pm 45^{\circ}, n=70$, median DSI $=0.26 ; p=0.35$, Mann-Whitney test).

\section{Binocular response properties in young animals}

One potential explanation for the reduction in disparity selectivity by MD is that disparity selectivity emerges over the period in which the deprivation occurred, and we have disrupted this 


\section{Normal Post-CP}

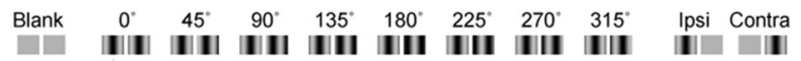

A

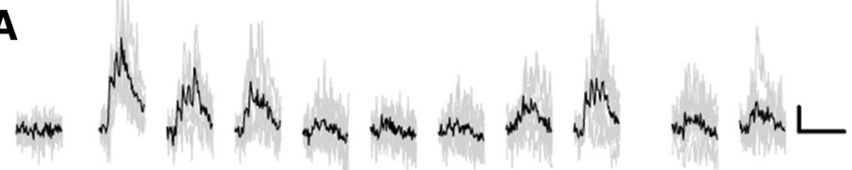

B

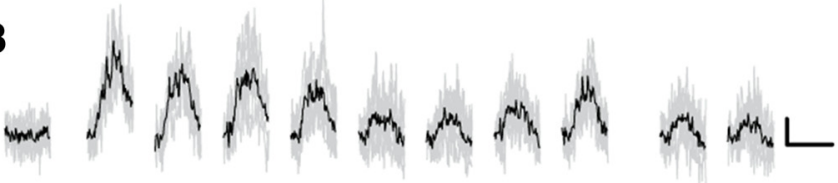

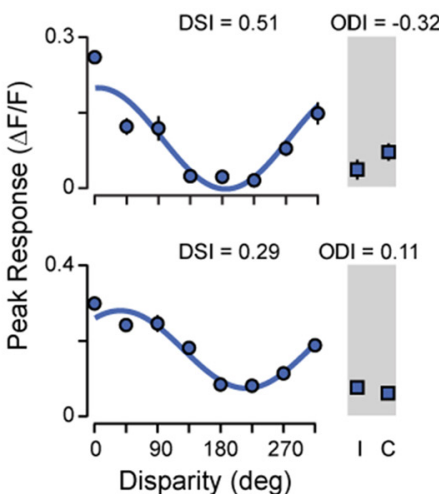

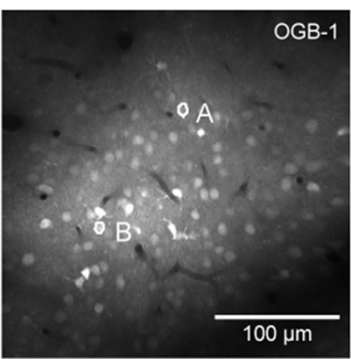

\section{4-Day Monocular Deprivation}

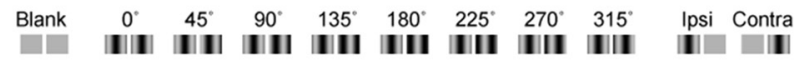

C

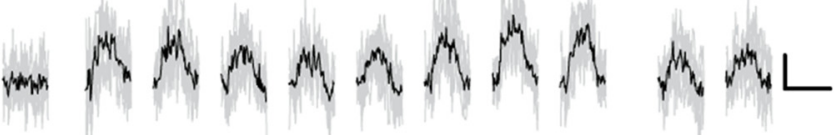

D

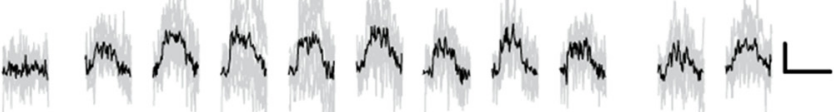

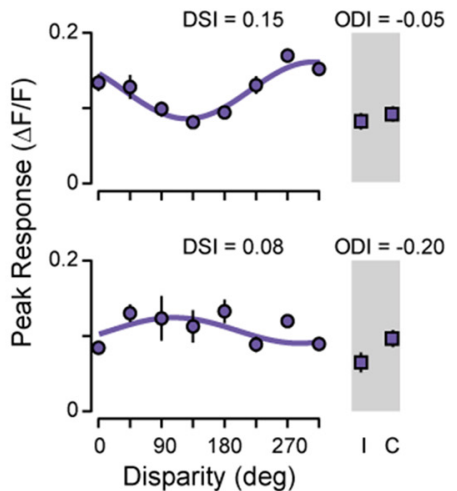

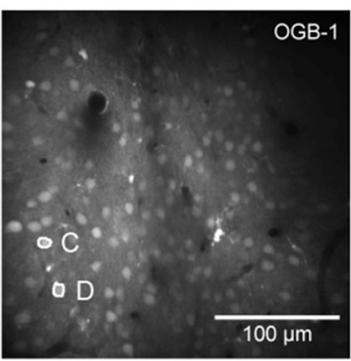

E
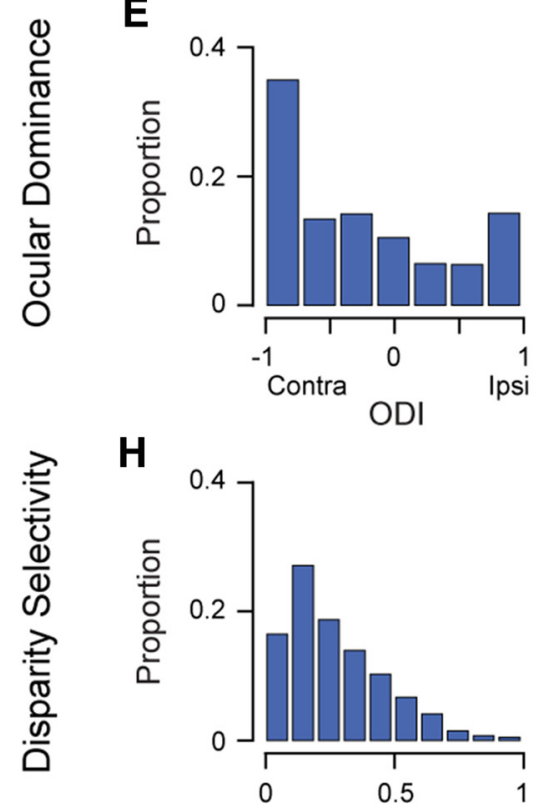

$\mathbf{F}$

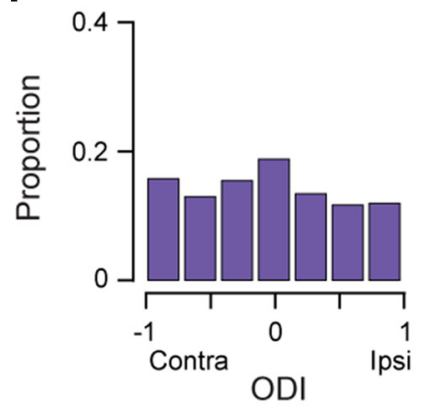

I

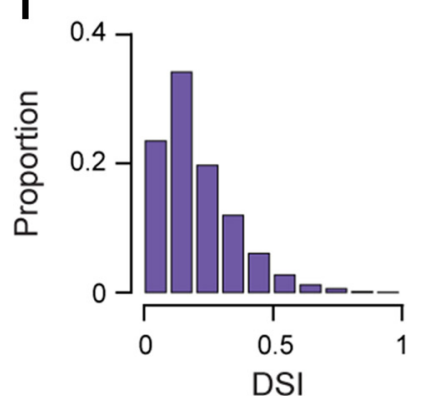

G

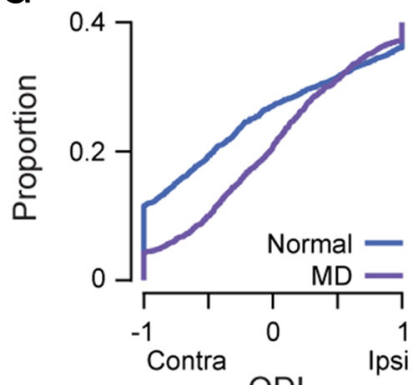

J

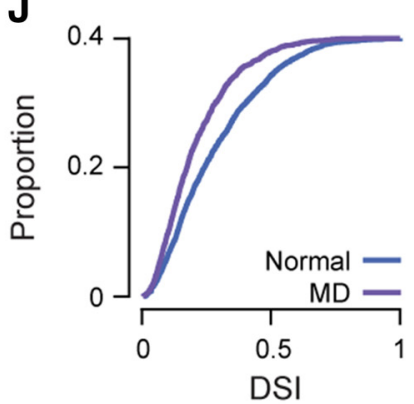

Figure 2. Two-photon imaging of binocular disparity selectivity in neurons from mouse V1 binocular zone. $\boldsymbol{A}-\boldsymbol{D}$, Example of calcium responses in a binocular neuron evoked by a range of binocular disparities ( 0 - 315 degrees), monocular stimulation of each eye, and a mean luminance screen. Gray represents individual traces. Black represents trial-average mean. Illustration of each stimulus shown above response traces. Scale bar indicates $10 \%$ change in fluorescence $(\Delta F / F)$ and $2 \mathrm{~s}$ duration. Mean $\pm \mathrm{SE}$ of peak $\Delta \mathrm{F} / \mathrm{F}$ shown in a tuning curve. Two-photon images (right) show fluorescence from OGB-1 AM dye. Drifting gratings used to measure these responses had a spatial frequency of 0.02 cycles/degree. $A, B$, Example neurons from a normal animal with different ocular preferences, but both selective to binocular disparity. $C, D$, Example neurons from an animal with $4 \mathrm{~d}$ MD during the CP. $E$, Population OD distribution from normal animals. $\boldsymbol{F}$, Population OD distribution from animals with $4 \mathrm{~d} \mathrm{MD.} \mathrm{G}$, Cumulative $0 \mathrm{D}$ distributions from normal and $\mathrm{MD}$ animals showing the shift toward binocularity in the MD animals. $\boldsymbol{H}$, Population distribution of DSI for cells from normal animals. I, Population distribution of DSI for cells from animals with MD. J, Cumulative distributions for DSI from normal and MD animals showing the shift toward lowed disparity selectivity following MD. 


\section{Normal Pre-CP}
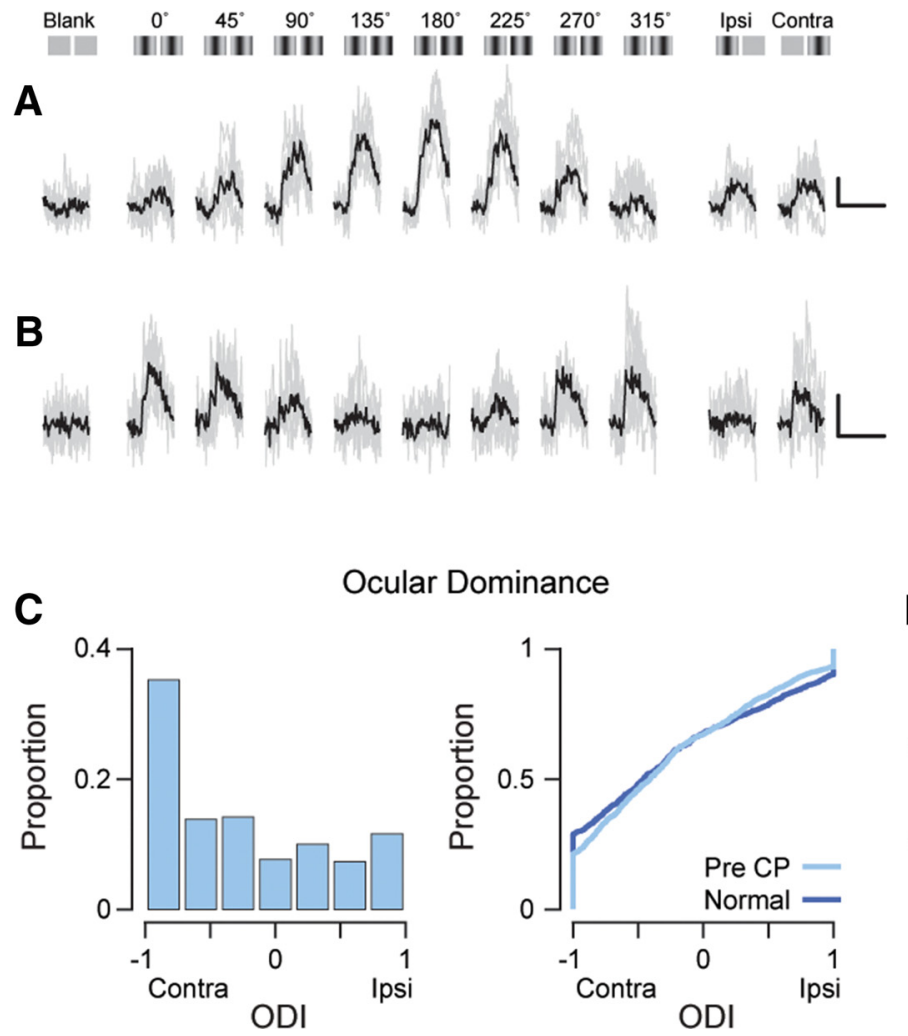

Ocular Dominance

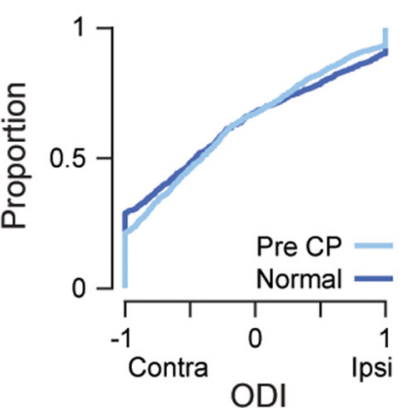

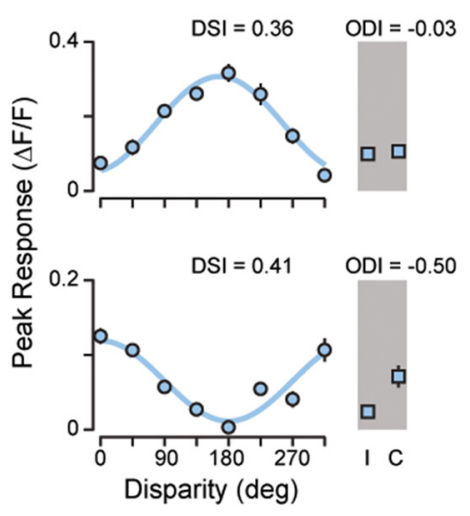

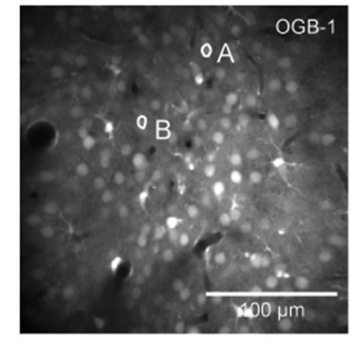

Disparity Selectivity

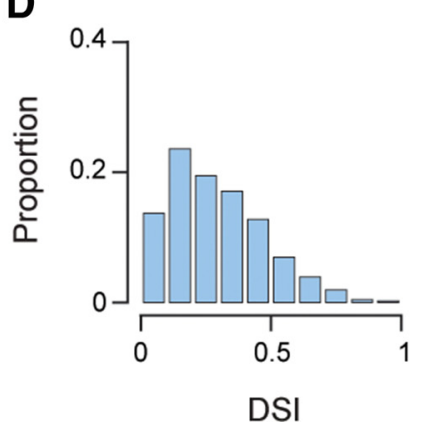

Figure 3. Disparity selectivity formed before the CP. $A, B$, Example tuning responses from neurons in an animal imaged before the CP. Both binocular and monocular neurons, as defined by $0 D$, are sensitive for binocular disparity. Drifting gratings used to measure these responses had a spatial frequency of 0.02 cycles/degree. C, $0 D$ distribution from animals before the $C P$ (left). Cumulative distributions from pre-CP and post-CP animals show similar OD (right). D, Distribution for DSI from animals before the CP (left). Cumulative distributions from pre-CP and post-CP animals show identical disparity selectivity (right).

development. To address this possibility, we measured binocular response properties of $\mathrm{V} 1$ neurons in mice during the epoch immediately before we performed MD (P25-P27). We denote these animals pre-CP, even though it has been shown that mouse V1 neurons are plastic at this age.

Across all animals used $(n=4$, P25-P27), we identified a total of 4137 neurons, of which 1197 neurons were visually responsive (for criterion, see Materials and Methods) for monocular stimuli (29\%) and 1685 neurons were visually responsive for binocular stimuli $(41 \%)$. As in normal animals, neurons responding to stimulation of both eyes equally $(\mathrm{ODI}=-0.03$; Fig. $3 A$ ) as well as those driven predominately by one eye (ODI $=-0.50$; Fig. $3 B$ ), were strongly modulated by binocular disparities (DSI $=0.36$ and DSI $=0.41$, respectively). The distribution of ODI and absolute value of the ODI in pre-CP neurons was identical to that of normal animals (pre-CP: median $|\mathrm{ODI}|=0.67$, mean $|\mathrm{ODI}|=$ $0.64 \pm 0.32, n=1197$; post-CP: median $|\mathrm{ODI}|=0.70$, mean $|\mathrm{ODI}|=0.64 \pm 0.34, n=1191 ; p=0.001$, Mann-Whitney test; Fig. 3C). Importantly, disparity selectivity, which reflects the underlying binocular synaptic integration between pre-CP and normal animals was similar, although pre-CP neurons showed slightly greater selectivity (pre-CP: median DSI $=0.26$, mean DSI $=0.29 \pm 0.18, n=1685$; post-CP: median DSI $=0.23$, mean DSI $=0.28 \pm 0.19, n=2505 ; p<0.01$, Mann-Whitney test; Fig. $3 D)$. These data suggest that disparity selectivity has formed before the $\mathrm{MD}$, and that the deprivation disrupted normal binocular integration.
Specificity of disparity selectivity loss in deprived animals

Despite a large shift in OD and disparity selectivity following MD, the impact of MD on OD and disparity selectivity was variable. For example, some neurons following MD maintain a strong preference for the contralateral eye (Fig. $2 F$ ), whereas others maintain a degree of disparity selectivity like that found in normal animals (Fig. 2I). This maintenance of selectivity diversity may result from differential impacts of MD. If so, then neurons with maintained OD preference should exhibit little change in disparity selectivity. Likewise, neurons receiving more input from the ipsilateral (nondeprived) eye should exhibit greater reductions in disparity selectivity. Thus, we grouped the neurons based on OD and compared their disparity selectivity (normal: $n=1012$, deprived: 838). Neurons maintaining preference for contralateral eye input following $\mathrm{MD}$ have little change in disparity selectivity (Fig. 4, unfilled circles; OD $<-0.6$ : $p=0.14$, Mann-Whitney test). All other OD groups showed significant reductions in disparity selectivity (Fig. 4 , filled circles; $p<0.01$, Mann-Whitney test). These results indicate that the circuit changes following MD are responsible for the reduction in disparity selectivity. We also examined the relationship between OD and disparity selectivity in pre-CP animals ( $n=910$ cells), finding similar degrees of disparity selectivity as in normal animals for neurons receiving contralateral input (OD $<0$ : $p=0.12$, Mann-Whitney test), but greater disparity selectivity for neurons receiving ipsilateral input $(\mathrm{OD}>0: p<0.01$, Mann-Whitney test). In both groups, we noticed that monocular neurons receiv- 


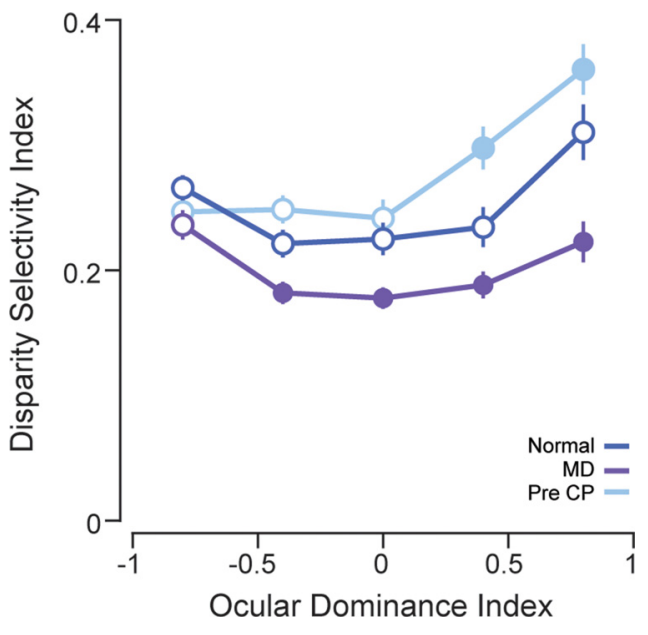

Figure 4. Decreased disparity selectivity selectively found in binocular and ipsilateral dominant neurons. Disparity selectivity for normal pre-CP (light blue), normal post-CP (dark blue), and deprived animals (purple) are shown for different $\mathrm{OD}$ groups. Each point indicates mean \pm SE. Filled circles represent significantly different values compared with normal post-CP animals.

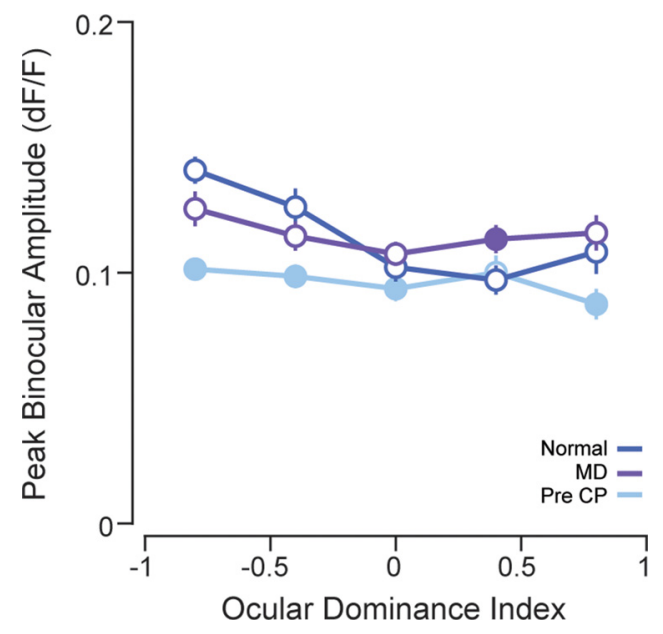

Figure 5. Response amplitude for preferred binocular disparity. Magnitude of calcium responses to the preferred binocular disparity for cells from normal pre-CP (light blue), normal post-CP (dark blue), and deprived animals (purple), shown for different $0 D$ groups. Each point indicates mean $\pm S E$. Filled circles represent significantly different values compared with normal post-CP animals.

ing ipsilateral input had greater disparity selectivity than those receiving contralateral input (pre-CP: $\mathrm{OD}<-0.6$ : median DSI $=0.20$, OD $>0.6$ : median DSI $=0.41, p<0.001$, MannWhitney test; Normal: OD $<-0.6$ : median DSI $=0.24$, OD $>0.6$ : median DSI $=0.34, p<0.001$, Mann-Whitney test). This is surprising given the strong contralateral bias typically found in mouse V1 (Figs. 2E, 3C) but suggests that the ipsilateral eye input prevails in controlling the degree of disparity selectivity.

The decline in disparity selectivity following MD may be the consequence of a change in overall activation of neurons by binocular stimulation or by a change in the degree in which cells are modulated by binocular stimulation. To address this, we compared fluorescence response amplitudes of neurons to binocular stimulation in normal animals, deprived animals, and pre-CP animals. We grouped neurons by OD and examined only disparity selective neurons (DSI >0.1) (Fig. 5). Except for ipsilateralbiased neurons $(0.2<\mathrm{OD}<0.6)$, there were no differences in response amplitude to the preferred binocular stimulus between normal and deprived animals ( $p>0.15$, Mann-Whitney test). We did find that pre-CP animals exhibited weaker preferred disparity responses, compared with post-CP and deprived animals ( $p<0.02$, Mann-Whitney for all groups, except ipsilaterally biased). This was also reflected in the average preferred binocular disparity response amplitude across all cells (normal post-CP = $0.13 \pm 0.08 ; \mathrm{MD}=0.12 \pm 0.06$; normal pre-CP $=0.10 \pm 0.05$; $\Delta \mathrm{F} / \mathrm{F})$. An identical trend was found when comparing the mean binocular response across all cells without excluding unselective cells (normal post-CP $=0.07 \pm 0.04$; $\mathrm{MD}=0.07 \pm 0.03$; pre$\mathrm{CP}=0.5 \pm 0.03 ; \Delta \mathrm{F} / \mathrm{F})$. These results suggest that the loss of disparity selectivity following MD cannot be attributed to a strong change in monocular or binocular response amplitudes, particularly because neurons receiving ipsilateral input are shown to have equivalent response amplitudes to binocular stimulation.

Our working model postulates that changes in the receptive field properties of ipsilateral inputs could underlie the loss of binocular integration following MD. One possibility is that the ipsilateral receptive fields lose spatial acuity, potentially through aberrant synaptic plasticity during MD. We tested this prediction by measuring the spatial frequency selectivity of individual neurons in normal and deprived animals. Using a vertical drifting grating, we first analyzed the responses of individual neurons to characterize their SF tuning (Fig. 6). Individual neurons responding to ipsilateral visual stimulation in normal animals had a broad range of spatial frequency preferences that extended to high spatial frequencies for the mouse visual system (Niell and Stryker, 2008), whereas ipsilateral neurons in animals with MD often responded to the lower spatial frequencies used (Fig. 6A). These examples followed the global trend across our population of cells, where we found a decrease in spatial resolution (high spatial frequency cutoff) caused by MD for cells responding to ipsilateral stimulation (normal: geometric mean resolution $=$ $0.07 \pm 0.05$ cycles/degree, $n=70 ; \mathrm{MD}$ : mean resolution $=0.05 \pm$ 0.03 cycles/degree, $n=117 ; p=0.02$, Mann-Whitney test; Fig. $6 \mathrm{~B}$, right). In contrast, we found no difference for contralaterally dominated neurons (normal: mean resolution $=0.10 \pm 0.04$ cycles/degree, $n=146 ; \mathrm{MD}$ : mean resolution $=0.09 \pm 0.04$ cycles/degree, $p=0.35, n=133$; Fig. $6 B$, left). These trends were also observed in the spatial frequency preferences of neurons following MD (Fig. 6C). The geometric mean peak spatial frequency for the ipsilateral eye was lower than that found in normal animals (MD geometric mean $=0.024 \pm 0.01, n=117$; normal geometric mean $=0.03 \pm 0.03, n=70 ; p=0.01$, unpaired $t$ test). In the subset of neurons for which we could compare the spatial frequency preference between the two eyes, there was little difference for normal animals (contralateral-ipsilateral preference, -0.16 octaves, $p=0.6$ ), but a much larger difference for MD animals (contralateral-ipsilateral preference, 0.62 octaves, $p<$ $0.05)$. These data indicate that ipsilateral receptive fields have been altered by MD, shifting toward lower spatial resolution for the nondeprived eye. Such a mismatch in receptive field parameters is consistent with the reduction in disparity selectivity we uncovered following MD.

\section{Discussion}

Experience-dependent plasticity during the $\mathrm{CP}$ guides maturation of sensory cortical circuits, both in anatomical and functional response properties of individual neurons. Neurons in the mouse V1 binocular zone shift their preference toward the ipsilateral eye if the contralateral eye is occluded during the $\mathrm{CP}$, causing increased binocularity as measured by OD (Dräger, 1975; Wagor et al., 1980; Gordon and Stryker, 1996; Hanover et al., 

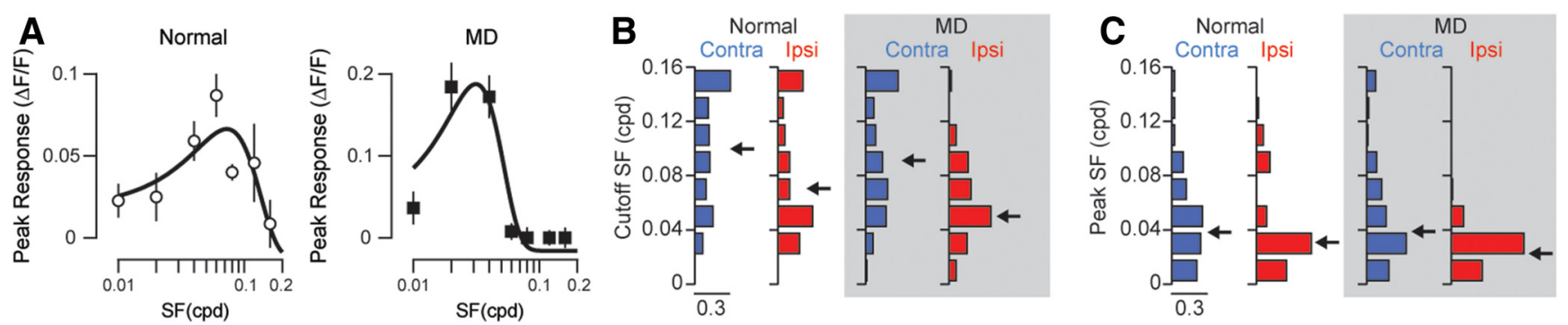

Figure 6. Loss of spatial acuity in the nondeprived eye. $\boldsymbol{A}$, Spatial frequency tuning of example neurons from normal (left, open circles) and deprived (right, filled squares) animals. Responses shown here are for ipsilateral eye visual stimulation with a vertical grating. Mean $\pm \mathrm{SE}$ of peak $\Delta \mathrm{F} / \mathrm{F}$ along with fits for spatial frequency selectivity (see Materials and Methods). $\boldsymbol{B}$, The spatial resolution for the contralateral (blue) and ipsilateral (red) eye stimulation. Histograms of the spatial resolution are shown for both normal animals as well as animals following MD (gray background). Black arrows indicate geometric mean. C, The peak spatial frequency for the contralateral (blue) and ipsilateral (red) eye stimulation. Histograms of the preferred spatial frequency are shown for both normal animals as well as animals following MD (gray background). Black arrows indicate geometric mean.

1999; Tagawa et al., 2005; Mrsic-Flogel et al., 2007; Hofer et al., 2009). We assessed how this increased binocularity in feedforward input transforms ocular integration as measured by binocular disparity selectivity using dichoptic stimulation of mouse V1 neurons in the binocular zone (Scholl et al., 2013a, 2015). We hypothesized three possible outcomes: increased disparity tuning from enhancement of nondeprived excitatory inputs, maintenance of disparity tuning through proportional changes between each eye (e.g., no change), or decreased tuning resulting from nondeprived eye inputs with mismatched spatial-temporal profiles. Using two-photon calcium imaging, we measured OD and the DSI in populations of neurons from normal, deprived, and normal pre-CP animals. Increased binocularity, evident in an OD shift, was accompanied by a decrease in DSI in animals that underwent contralateral MD. Further, neurons receiving input from the ipsilateral (nondeprived) eye displayed the greatest loss of selectivity, and a loss of spatial frequency sensitivity. In comparing pre-CP and post-CP animals, we observe no difference in DSI distributions, demonstrating that MD was not impairing normal development of disparity selectivity. Our data suggest OD plasticity-mediated enhancement of binocularity drives a misalignment of synaptic inputs from the two eyes disrupting existing binocular integration (Fig. $1 B$, right).

How can we reconcile the paradoxical increase in the population of neurons receiving input from both eyes with decreased disparity selectivity? We propose that the specificity of spatial inputs coming from each eye is disrupted, and that this disruption leads to a decrease in disparity selectivity. It is known that MD causes changes in the synaptic connectivity within V1, and those changes could lead to a reduction in the spatial alignment responsible for disparity selectivity (Antonini et al., 1999). Contralateral MD should increase the number of ipsilateral eye inputs projecting to individual neurons, but their spatial receptive field may be distinct. The additional ipsilateral inputs would create a more diffuse receptive field following MD. This mismatch in the spatial dispersion of the receptive fields should lead to weaker disparity selectivity as there is no longer a narrow set of spatial disparities that lead to binocular summation. In concert with these changes in disparity selectivity, the increased ipsilateral inputs cause neuronal responses become more binocular following MD (Fig. 2G).

Disparity selectivity, like orientation and direction selectivity, appears to be another receptive field property emerging early in development (Chino et al., 1997; Feller and Scanziani, 2005; White et al., 2007), as we find little or no difference between pre-CP and post-CP animals (Fig. 3). It is currently not known how early binocular disparity selectivity emerges or the exact role visual experience plays in sculpting its formation, although recent work from Gu and Cang (2016) suggests binocular matching feedforward thalamic input onto individual neurons in pre-CP animals could provide the necessary signals. In carnivores and primates, little or no disparity sensitivity is found shortly after eye opening, but this selectivity is enhanced within a few weeks of visual experience (Pettigrew, 1974; Freeman and Ohzawa, 1992; Chino et al., 1997). Interestingly, newborn lambs do exhibit modest disparity selectivity (Ramachandran et al., 1977). Thus, it is possible that disparity selectivity is developed starting near eye opening in mice, and it is close to matured near the normal CP. A potential period of time for this maturation would be during the matching of orientation preference between the two eyes (P20P23) (Wang et al., 2010; Sarnaik et al., 2014). Our measurements were done at a slightly later age (P25-P27) and may therefore miss the important developmental period for binocular integration in mouse V1.

In carnivores and primates, the effects of MD on binocularity, as assayed by OD or disparity selectivity, reflect shifts in the same direction. There are declines in both measurements: most neurons respond only to the open eye under monocular conditions, and disparity selectivity is dramatically reduced (Sclar et al., 1986; Freeman and Ohzawa, 1988; Vorobyov et al., 2007). We took advantage that MD causes a paradoxical increase in binocularity by OD in mice to determine whether these two functional properties are linked. We find that OD and disparity selectivity, two measures of binocularity, shift in opposite directions in mice, indicating that these two properties are not linked. Previous developmental work in carnivores and primates has indicated that $\mathrm{MD}$ affects $\mathrm{OD}$ and disparity selectivity to different degrees. In conditions of partial MD, in which some binocular experience is allowed everyday, disparity selectivity strongly declines, whereas the monocular responses of neurons, including contrast sensitivity and OD, are only mildly affected (Sakai et al., 2006; Schwarzkopf et al., 2007; Vorobyov et al., 2007).

The relationship between OD and disparity selectivity is important to guide ongoing efforts to recover normal stereopsis in people with amblyopia. Brief MD in people increases the perceptual strength of the deprived eye (Lunghi et al., 2011; Zhou et al., $2013 \mathrm{a}, \mathrm{b})$ and the strength of evoked potentials associated with cortical processing (Lunghi et al., 2015). These results raise an important question of whether MD, or partial occlusion, could be used in adults with amblyopia to assist a recovery of binocular function (Zhou et al., 2013c; Hess and Thompson, 2015). Indeed, Hess and Thompson (2015) have demonstrated that changes in the strength of input from the weak eye are possible with brief periods of deprivation in people with amblyopia. It is not clear, 
however, whether these changes in the input strength driven by deprivation will induce the emergence of stereopsis, although strengthening the weakened eye may be the first step to recovering normal binocular function.

\section{References}

Antonini A, Fagiolini M, Stryker MP (1999) Anatomical correlates of functional plasticity in mouse visual cortex. J Neurosci 19:4388-4406. Medline

Brainard DH (1997) The psychophysics toolbox. Spat Vis 10:433-436. CrossRef Medline

Cang J, Rentería RC, Kaneko M, Liu X, Copenhagen DR, Stryker MP (2005) Development of precise maps in visual cortex requires patterned spontaneous activity in the retina. Neuron 48:797-809. CrossRef Medline

Carandini M, Ferster D (2000) Membrane potential and firing rate in cat primary visual cortex. J Neurosci 20:470-484. Medline

Chino YM, Smith EL 3rd, Hatta S, Cheng H (1997) Postnatal development of binocular disparity sensitivity in neurons of the primate visual cortex. J Neurosci 17:296-307. Medline

Cumming BG, Parker AJ (1999) Binocular neurons in V1 of awake monkeys are selective for absolute, not relative, disparity. J Neurosci 19:5602-5618. Medline

DeAngelis GC, Ohzawa I, Freeman RD (1993) Spatiotemporal organization of simple-cell receptive fields in cat's striate cortex: II. Linearity of temporal and spatial summation. J Neurophysiol 69:1118-1135. Medline

DeAngelis GC, Ohzawa I, Freeman RD (1995) Neuronal mechanisms underlying stereopsis: how do simple cells in the visual cortex encode binocular disparity? Perception 24:3-31. CrossRef Medline

Dräger UC (1975) Receptive fields of single cells and topography in mouse visual cortex. J Comp Neurol 160:269-290. CrossRef Medline

Espinosa JS, Stryker MP (2012) Development and plasticity of the primary visual cortex. Neuron 75:230-249. CrossRef Medline

Feller MB, Scanziani M (2005) A pre-CP for plasticity in visual cortex. Curr Opin Neurobiol 15:94-100. CrossRef Medline

Freeman RD, Ohzawa I (1988) Monocularly deprived cats: binocular tests of cortical cells reveal functional connections from the deprived eye. J Neurosci 8:2491-2506. Medline

Freeman RD, Ohzawa I (1992) Development of binocular vision in the kitten's striate cortex. J Neurosci 12:4721-4736. Medline

Garaschuk O, Milos RI, Konnerth A (2006) Targeted bulk-loading of fluorescent indicators for two-photon brain imaging in vivo. Nat Protoc 1:380-386. CrossRef Medline

Golshani P, Portera-Cailliau C (2008) In vivo 2-photon calcium imaging in layer 2/3 of mice. J Vis Exp 13:681. CrossRef Medline

Gordon JA, Stryker MP (1996) Experience-dependent plasticity of binocular responses in the primary visual cortex of the mouse. J Neurosci 16: 3274-3286. Medline

Gu Y, Cang J (2016) Binocular matching of thalamocortical and intracortical circuits in the mouse visual cortex. eLife 5:e22032. CrossRef Medline

Hanover JL, Huang ZJ, Tonegawa S, Stryker MP (1999) Brain-derived neurotrophic factor overexpression induces precocious critical period in mouse visual cortex. J Neurosci 19:RC40. Medline

Hess RF, Thompson B (2015) Amblyopia and the binocular approach to its therapy. Vision Res 114:4-16. CrossRef Medline

Hofer SB, Mrsic-Flogel TD, Bonhoeffer T, Hübener M (2009) Experience leaves a lasting structural trace in cortical circuits. Nature 457:313-317. CrossRef Medline

Hubel DH, Wiesel TN (1962) Receptive fields, binocular interaction and functional architecture in the cat's visual cortex. J Physiol 160:106-154. CrossRef Medline

Joshua DE, Bishop PO (1970) Binocular single vision and depth discrimination: receptive field disparities for central and peripheral vision and binocular interaction on peripheral single units in cat. Exp Brain Res 10:389-416. CrossRef Medline

Julesz B (1971) Foundations of cyclopean perception. Chicago: University of Chicago.

Kameyama K, Sohya K, Ebina T, Fukuda A, Yanagawa Y, Tsumoto T (2010) Difference in binocularity and ocular dominance plasticity between GABAergic and excitatory cortical neurons. J Neurorsci 30:1551-1559. CrossRef Medline

Kara P, Boyd JD (2009) A micro-architecture for binocular disparity and ocular dominance in visual cortex. Nature 458:627-631. CrossRef Medline
Katz LC, Crowley JC (2002) Development of cortical circuits: lessons from ocular dominance columns. Nat Rev Neurosci 3:34-42. CrossRef Medline

Kerr JN, Greenberg D, Helmchen F (2005) Imaging input and output of neocortical networks in vivo. Proc Natl Acad Sci U S A 102:14063-14068. CrossRef Medline

LeVay S, Voigt T (1988) Ocular dominance and disparity coding in cat visual cortex. Vis Neurosci 1:395-414. CrossRef Medline

Longordo F, To MS, Ikeda K, Stuart GJ (2013) Sublinear integration underlies binocular processing in primary visual cortex. Nat Neurosci 16:714723. CrossRef Medline

Lunghi C, Burr DC, Morrone C (2011) Brief periods of monocular deprivation disrupt ocular balance in human adult visual cortex. Curr Biol 21: R538-R539. CrossRef Medline

Lunghi C, Berchicci M, Morrone C, Russo FD (2015) Short-term monocular deprivation alters early components of visual evoked potentials. J Physiol 19:4361-4372. CrossRef Medline

Métin C, Godement P, Imbert M (1988) The primary visual cortex in the mouse: receptive field properties and functional organization. Exp Brain Res 69:594-612. Medline

Mrsic-Flogel TD, Hofer SB, Ohki K, Reid RC, Bonhoeffer T, Hübener M (2007) Homeostatic regulation of eye-specific responses in visual cortex during ocular dominance plasticity. Neuron 54:961-972. CrossRef Medline

Nauhaus I, Nielsen KJ, Callaway EM (2012) Nonlinearity of two-photon $\mathrm{Ca}^{2+}$ imaging yields distorted measurements of tuning for V1 neuronal populations. J Neurophysiol 107:923-936. CrossRef Medline

Niell CM, Stryker MP (2008) Highly selective receptive fields in mouse visual cortex. J Neurosci 28:7520-7536. CrossRef Medline

Ohki K, Chung S, Ch'ng YH, Kara P, Reid RC (2005) Functional imaging with cellular resolution reveals precise micro-architecture in visual cortex. Nature 433:597-603. CrossRef Medline

Ohzawa I, Freeman RD (1986) The binocular organization of simple cells in the cat's visual cortex. J Neurophysiol 56:221-242. Medline

Pelli DG (1997) The VideoToolbox software for visual psychophysics: transforming numbers into movies. Spat Vis 10:437-442. CrossRef Medline

Pettigrew JD (1974) The effect of visual experience on the development of stimulus specificity by kitten cortical neurones. J Physiol 237:49-74. CrossRef Medline

Pettigrew JD, Nikara T, Bishop PO (1968) Binocular interaction on single units in cat striate cortex: simultaneous stimulation by single moving slit with receptive fields in correspondence. Exp Brain Res 6:391-410. Medline

Poggio GF, Fischer B (1977) Binocular interaction and depth sensitivity in striate and prestriate cortex of behaving rhesus monkey. J Neurophysiol 40:1392-1405. Medline

Priebe NJ (2008) The relationship between subthreshold and suprathreshold ocular dominance in cat primary visual cortex. J Neurosci 28:85538559. CrossRef Medline

Ramachandran VS, Clarke PG, Whitteridge D (1977) Cells selective to binocular disparity in the cortex of newborn lambs. Nature 268:333-335. CrossRef Medline

Ringach DL, Shapley RM, Hawken MJ (2002) Orientation selectivity in macaque V1: diversity and laminar dependence. J Neurosci 22:5639-5651. Medline

Sakai E, Bi H, Maruko I, Zhang B, Zheng J, Wensveen J, Harwerth RS, Smith EL 3rd, Chino YM (2006) Cortical effects of brief daily periods of unrestricted vision during early monocular form deprivation. J Neurophysiol 95:2856-2865. CrossRef Medline

Sarnaik R, Wang BS, Cang J (2014) Experience dependent and independent binocular correspondence of receptive field subregions in mouse visual cortex. Cereb Cortex 24:1658-1670. CrossRef Medline

Scholl B, Burge J, Priebe NJ (2013a) Binocular integration and disparity selectivity in mouse primary visual cortex. J Neurophysiol 109:30133024. CrossRef Medline

Scholl B, Tan AY, Priebe NJ (2013b) Strabismus disrupts binocular synaptic integration in primary visual cortex. J Neurosci 33:17108-17122. CrossRef Medline

Scholl B, Pattadkal JJ, Dilly GA, Priebe NJ, Zemelman BV (2015) Local integration accounts for weak selectivity of mouse neocortical parvalbumin interneurons. Neuron 87:424-436. CrossRef Medline

Schwarzkopf DS, Vorobyov V, Mitchell DE, Sengpiel F (2007) Brief daily 
binocular vision prevents monocular deprivation effects in visual cortex. Eur J Neurosci 25:270-280. CrossRef Medline

Sclar G, Ohzawa I, Freeman RD (1986) Binocular summation in normal, monocularly deprived and strabismic cats: visual evoked potentials. Exp Brain Res 62:1-10. Medline

Stosiek C, Garaschuk O, Holthoff K, Konnerth A (2003) In vivo two-photon calcium imaging of neuronal networks. Proc Natl Acad Sci U S A 100: 7319-7324. CrossRef Medline

Tagawa Y, Kanold PO, Majdan M, Shatz CJ (2005) Multiple periods of functional ocular dominance plasticity in mouse visual cortex. Nat Neurosci 8:380-388. CrossRef Medline

Tan AY, Brown BD, Scholl B, Mohanty D, Priebe NJ (2011) Orientation selectivity of synaptic input to neurons in mouse and cat primary visual cortex. J Neurosci 31:12339-12350. CrossRef Medline

Van den Bergh G, Zhang B, Arckens L, Chino YM (2010) Receptive-field properties of V1 and V2 neurons in mice and macaque monkeys. J Comp Neurol 518:2051-2070. CrossRef Medline

Vorobyov V, Schwarzkopf DS, Mitchell DE, Sengpiel F (2007) Monocular deprivation reduces reliability of visual cortical responses to binocular disparity stimuli. Eur J Neurosci 26:3553-3563. CrossRef Medline

Wagor E, Mangini NJ, Pearlman AL (1980) Retinotopic organization of striate and extrastriate visual cortex in the mouse. J Comp Neurol 193: 187-202. CrossRef Medline

Wang BS, Sarnaik R, Cang J (2010) Critical period plasticity matches binocular orientation preference in the visual cortex. Neuron 65:246-256. CrossRef Medline

White LE, Fitzpatrick D (2007) Vision and cortical map development. Neuron 56:327-338. CrossRef Medline

Zhou J, Clavagnier S, Hess RF (2013a) Short-term monocular deprivation strengthens the patched eye's contribution to binocular combination. J Vis 13:12. CrossRef Medline

Zhou J, Thompson B, Hess RF (2013b) A new form of rapid binocular plasticity in adult with amblyopia. Sci Rep 3:2638. CrossRef Medline

Zhou J, Jia W, Huang CB, Hess RF (2013c) The effect of unilateral mean luminance on binocular combination in normal and amblyopic vision. Sci Rep 3:2012. CrossRef Medline 